\title{
Lessons Learned Through the Journey of a Medical Toxicologist While Characterizing Lead Hazards in the Republic of Georgia
}

\author{
Ziad Kazzi $^{1}$ (1) $\cdot$ Levan Gabelaia $^{2} \cdot$ Lela Shengelia $^{2} \cdot$ Lela Sturua $^{2} \cdot$ Bret Ericson $^{3} \cdot$ Anni Giorgobiani $^{4}$. \\ Andria Nadiradze ${ }^{5}$. Amiran Gamkrelidze ${ }^{2}$
}

Received: 10 October 2019 / Revised: 13 October 2019 / Accepted: 15 October 2019 / Published online: 14 November 2019

(C) American College of Medical Toxicology 2019

Keywords Lead $\cdot$ Georgia $($ Republic) $\cdot$ United Nations $\cdot$ Child

Between September and December 2018, the Georgian Ministry of Internally Displaced Persons from the Occupied Territories, Labor, Health and Social Affairs and the United Nations International Children's Emergency Fund (UNICEF) conducted a study that screened children aged 2-7 years for lead exposure. The study investigators obtained the venous blood sample during the UNICEF Multiple Indicator Cluster Survey (MICS) that was being performed in Georgia. UNICEF had previously conducted the MICS study over 300 times in more than 117 countries and had produced data on key indicators on the well-being of children and women in order to guide policies that improve their health [1]. The MICS data contributed to the identification of the Millennium Development Goals (MDG) indicators. It will again serve as a data source during the 2030 Sustainable Development Initiative to assess Sustainable Development Goals (SDGs) indicators.

The traditional MICS study does not include data on blood lead levels (BLLs). A lead exposure assessment was added to this survey at the request of the Georgian government based

Supervising Editor: Mark B. Mycyk, MD

Ziad Kazzi

zkazzi@emory.edu

1 Emory University, 50 Hurt Plz SE Suite 600, Atlanta, GA 30303, USA

2 National Center for Disease Control and Public Health, 4 Kakheti Hwy, Tbilisi, Georgia

3 Pure Earth, 475 Riverside Drive, Suite 860, New York, NY 10115, USA

4 Iashvili Children's Central Hospital in Tbilisi, Mgaloblishvili Str, 13 Tbilisi, Georgia

5 UNICEF Georgia, 9 Eristavi Str. UN House 4th Floor, 0179 Tbilisi, Georgia on the previous pilot studies that had been conducted by the Georgian National Center for Disease Control and Public Health (NCDC) in collaboration with Emory University and the Iashvili Children's Central Hospital in Tbilisi. This collaborative work started with a cross-sectional pilot study between November and December 2015 using a convenience sample of children aged 2-5 years visiting the private, tertiary Iashvili Children Central Hospital in Tbilisi. Technical support was provided by the United States (US) Centers for Disease Control and Prevention (CDC). Venous BLLs were measured using a LeadCare II analyzer provided by Magellan Inc. In this study, 254 children were included and the mean BLL was 5.7 $\mathrm{mcg} / \mathrm{dl}$ (SD 5.3). The median BLL was $3.9 \mathrm{mcg} / \mathrm{dl}$ (range 3.357.0). Thirty-three percent of BLLs were $\geq 5 \mathrm{mcg} / \mathrm{dl}, 9.5 \% \geq$ $10 \mathrm{mcg} / \mathrm{dl}, 2.8 \% \geq 20 \mathrm{mcg} / \mathrm{dl}$, and $0.4 \% \geq 45 \mathrm{mcg} / \mathrm{dl}$. The research team assessed the home of the child who had the highest BLL in this cohort and identified high levels of lead in paint chips collected near the window seal $(0.902 \mathrm{wt} \%$, allowable amount in the US less than $0.0009 \%$ ). Soil and water samples collected from that home did not reveal elevated lead levels. The child was referred for medical evaluation and the family moved out of the residence. They have since been lost to follow-up. Another publication from 2005 had discussed a case series of elevated blood lead levels in members of a Georgian family living in the US who were consuming traditional spices brought back from Georgia [2].

On May 17, 2017, the US Food and Drug Administration issued a warning against using the Magellan Diagnostics LeadCare II analyzer with venous blood. This unexpected event produced concerns about the results of the initial study conducted in Tbilisi. In consequence, the investigators tested 100 children (age 1-9 years) attending the Iasvilli Children's Central Hospital in Tbilisi using venous blood and Inductively Coupled Plasma Mass Spectrometry (ICP-MS). According to this study, the median BLL was $4 \mathrm{mcg} / \mathrm{dl}$ (IQR 2, 8). Thirtyseven percent had BLLs $\geq 5 \mathrm{mcg} / \mathrm{dl}, 17 \%$ had a BLLs $\geq 10$ 
$\mathrm{mcg} / \mathrm{dl}, 2 \%$ had a BLLs $\geq 10 \mathrm{mcg} / \mathrm{dl}$, and $1 \%$ had BLLs $\geq 30$ $\mathrm{mcg} / \mathrm{dl}$ (Source: Unpublished data from the Georgian National Center for Disease Control).

Several planning meetings among various stakeholders and training courses for healthcare providers in 2014 and 2018 accompanied the research efforts that raised awareness of hazards of lead exposure among the medical and public health communities. Additionally, volunteers and non-governmental organizations identified lead contamination in 268 everyday items including toys, ceramics, and food from 17 families living in Tbilisi. These activities alerted the public to this hazard and resulted in social media attention and related communication.

In the MICS study, trained fieldwork teams conducted face-toface interviews with a national randomly selected sample of 1578 children on a variety of topics. The blood samples were obtained by trained phlebotomists and were analyzed by the Italian National Institute of Health laboratory using ICP-MS. This analysis revealed that $41 \%$ of children had BLLs $\geq 5 \mathrm{mcg} / \mathrm{dL}$, and $16 \%$ of children have BLLs $\geq 10 \mathrm{mcg} / \mathrm{dL}$. Twenty-five percent of children had BLLs between 5 and $10 \mathrm{mcg} / \mathrm{dL}$. The data analysis by region of the country revealed a higher prevalence of elevated BLLs in the western part of Georgia. For example, in the Adjara region, $85 \%$ of the children tested had a BLL $\geq 5 \mathrm{mcg} / \mathrm{dl}$. A comprehensive analysis of these results is forthcoming and will be included in the overall MICS report.

The Georgian government responded promptly to this public health hazard using multiple modalities and actions: a multi-agency committee under the leadership of the ministry of health and the NCDC was created with the goal of developing a national plan to mitigate lead hazards in the population. The potential sources of lead exposures were assessed using a phone-based survey of families enrolled in the MICS study. Additional training seminars and press releases were organized and the NCDC assessed the homes of the children with BLLs $\geq 30 \mathrm{mcg} / \mathrm{dl}$. This included a medical evaluation as well as an in-home assessment for possible sources of lead exposure. The results of these home evaluations are forthcoming and will be a component of the Health Promotion State Program, Environment and Health that will include public education campaigns regarding lead hazards.

Based on recommendations of the US Centers for Disease Control and Prevention and the American Academy of Pediatrics, the Ministry of Health approved a clinical protocol for the early identification and management of toxic effects of lead in children (National Environmental Health Action Plan 2018-2022 approved by the Government of Georgia on December 29, 2018). Additionally, all children participating in the survey, members of their families up to 18 years of age, and pregnant women will be tested, treated, and given relevant recommendations as per the protocol (Government of Georgia Order on "Early Identification of Toxic Effect of Lead in Children and Management Measures"). Children $\leq 18$ years who were not part of the survey will be tested and managed by their physician according to the long-term multi-sectoral program that is currently under development.

Although, the prevalence of BLLs $\geq 5 \mathrm{mcg} / \mathrm{dl}$ is higher than the corresponding current prevalence in the US, it is similar to the situation in the late 1980s [3]. In 2016, US data showed that the percent of children aged less than 3 years with a BLL $\geq 5 \mathrm{mcg} / \mathrm{dl}$ varied by state between 1 and $6 \%$. Comparatively, in 2012, US data showed that the percent of children aged $<3$ years with a BLL $\geq 5 \mathrm{mcg} / \mathrm{dl}$ varied by state between 1 and $17 \%$ (for the state of Louisiana). According to the US CDC, the prevalence of BLLs $\geq 10 \mathrm{mcg} / \mathrm{dL}$ among children aged 15 years decreased substantially, from $88.2 \%$ during the National Health and Nutrition Examination Survey II (NHANES) covering the 1976-1980 period to $8.9 \%$ during NHANES III, Phase 1 (1988-1994) [4].

The US experience with lead hazards has demonstrated that public health measures are successful over time in decreasing exposure in the population. A multidisciplinary team of public health professionals, medical toxicologists, governmental agencies, private hospitals, and non-governmental organizations is confronting the challenge from lead hazards in Georgia. A number of resources are needed to ensure the success of these efforts. There is a need for laboratory capabilities to support a long-term national surveillance. Additionally, the medical workforce competency in evaluating and managing individuals with elevated BLL should be enhanced with the support of medical toxicology experts. Environmental control policies and public awareness campaigns should also be implemented at national level. Although these necessary actions will likely take years to be completed, the journey of a thousand miles starts with a single step. We are optimistic that this journey will be successful through the existing collaborations that have shed light on this important public health problem in Georgia and beyond.

Acknowledgments The authors would like to thank the following collaborators:

- George Katsitadze, MD, American Clinic-Tbilisi, Tbilisi, Georgia

- Ghassan Khalil, PhD, UNICEF Georgia Office, Tbilisi, Georgia

- Tim Moran, PhD, Emory University, Atlanta, Georgia, USA

- Tako Ugulava, UNICEF Georgia Office, Tbilisi, Georgia

- Alessandro Alimonte, National Institute of Health, Italy

- Michael Kosnett, MD, MPH, University of Colorado at Denver, Denver, Colorado, USA

- Fred Henretig, MD, Children's Hospital of Philadelphia, Philadelphia, Pennsylvania, USA

The authors would like to thank the United States Embassy in Tbilisi, Georgia, and the United States Centers for Disease Control and Prevention for their technical support during this project.

Sources of Funding None

\section{Compliance with Ethical Standards}

Conflicts of Interest None 


\section{References}

1. About MICS. In: United Nations International Children's Emergency Fund (UNICEF) http://mics.unicef.org/about. Accessed April 252019.

2. Woolf AD, Woolf NT. Childhood lead poisoning in 2 families associated with spices used in food preparation. Pediatrics. 2005;116(2): e314-8.

3. Pirkle JL, Brody DJ, Gunter EW, Kramer RA, Paschal DC, Flegal $\mathrm{KM}$, et al. The decline in blood lead levels in the United States. The
National Health and Nutrition Examination Surveys (NHANES). JAMA. 1994 27;272(4):284-91.

4. Centers for Disease Control and Prevention. Blood lead levelsUnited States, 1988-1991. MMWR Morb Mortal Wkly Rep. 1994;43(30):545-8.

Publisher's Note Springer Nature remains neutral with regard to jurisdictional claims in published maps and institutional affiliations. 